\title{
Male Factor Infertility.
}

\author{
Sajida Sboui ${ }^{1}$, Ahmed Tabbabi ${ }^{2 *}$ \\ ${ }^{1}$ Faculty of Medicine of Monastir, Monastir University, Monastir, Tunisia \\ ${ }^{2}$ Department of Hygiene and Environmental Protection, Ministry of Public Health, Tunis, Tunisia
}

Accepted on February 19, 2018

\section{Short Communication}

A major public health problem, couple infertility represents a scientific, medical and financial challenge. However, the psychological impact of the whole procedure must not be neglected. Studies have concluded that $15 \%$ of couples have difficulties in procreation, with $2 / 3$ of the cases having an exclusive or associated male cause [1] and it is estimated that $10 \%$ is the frequency of male sterility phenotype in the human population [2]. Different causes may trigger male infertility including the obstruction of the genital tract, the erection or ejaculation problems, the endocrine problems (hypogonadotropic hypogonadism), the defects in spermatogenesis and the impaired function of mature spermatozoa. Recent advances in assisted reproductive technologies, including intracytoplasmic sperm injection (ICSI), have upset the prognosis of male infertility. Men, previously considered as permanently sterile, can now have offspring, raising the problem of the possible transmission of genetic abnormalities responsible for their infertility.

Molecular biology has made it possible to recover etiologies on a scale that has not been explored until now. It opened up new perspectives, but it also made us understand the extent of our shortcomings. Without questioning the explanatory value of certain recognized etiologies (varicocele, genitourinary infection, anti-spermatozoid antibodies, etc.), this profound change in the approach to male infertility has the consequence of adding a new dimension. However, if the role of researchers is important, what about that of clinicians who handle gametes, give hope to couples, without completely controlling the consequences. Indeed, medically assisted procreation, with ICSI, has made a huge difference in the treatment of male infertility. However, father-to-son vertical transmission of genetic abnormalities, such as the Y chromosome micro-deletion, could be very well, if they do not remain stable over generations, lead to lifethreatening pathologies. Thus, the couple must benefit from exhaustive information (genetic counseling) as to the potential risks allowing them to understand and assume their share of responsibility for the I.C.S.I. (informed consent). Indeed, thanks to techniques of medical assistance procreation (AMP), infertile men can now transmit a possible genetic abnormality to their offspring. Natural selection that prevented the transmission of mutations resulting in infertility is now being short-circuited by technology which exposes the risk of a future increase in the genetic causes of infertility. The identification of genetic factors is becoming a necessary step in the management of infertile couples and genetic counseling is now an essential step in the assessment of infertility [3].

The origin of these conditions can be anatomical, genetic, or related to the environment [4]. The relationship between the environment and infertility has been the subject of much work [5]. These studies have been particularly successful following the publication by several groups of disturbing results showing a significant decline in sperm quality during the second half of this century, and particularly in industrialized countries $[6,7]$. In addition, the existence of chromosomal abnormalities and familial cases shows that a genetic defect is at the origin of certain cases of sterility [8,9]. Among these, chromosomal abnormalities are the most frequent and require the study of the karyotype of patients with azoospermia or nonobstructive oligozoospermia. Thanks to advances in molecular biology, finer genetic anomalies have been described: micro-deletions of the Y chromosome have identified a part of the genes, located on the long arm of this chromosome, playing a role in spermatogenesis. The study of animal models has also led to the involvement of other genes that, in humans, are at the origin of sterility. The use of new pan genomic genotyping techniques has recently led to the implication of new genes in specific phenotypes of male infertility. Very recently, the genetic origin of two forms of severe teratozoospermia (morphological abnormalities of the spermatozoon) has been identified: in patients with spermatozoa, they are either macrorephalic or have a round head without acrosome (globozoospermia) [10-13]. Above all, they provide a better understanding of the fundamental mechanisms of spermatogenesis and may ultimately lead to therapeutic advances [14]. For the patients concerned, these advances make it possible to perform a diagnosis, to refine the prognosis and thus to better guide the management.

At present, the study of the genetics of male infertility is upset by the analysis of polymorphisms. Thus, some polymorphisms or variant genes involved in the proper course of spermatogenesis are considered as potential risk factors that may contribute to the severity of spermatogenic defect. Several variants were thus associated with the occurrence of male infertility. Nevertheless, these association studies are not always reproducible. This may result from various important parameters: the size and composition of the populations studied, particularly the control populations, the type of polymorphism studied and the techniques used to demonstrate it, the multifactorial conditions and the phenotypic heterogeneity of male infertility as well as ethnic and geographical differences that contribute to genetic variation. The phenotypic effects of gene polymorphisms are themselves modulated by other genetic factors or "genetic background" as well as by environmental factors. It is therefore likely that some polymorphisms cause an alteration of spermatogenesis or a lack of testicular function only in the presence of a particular genetic background and/or in the presence of certain environmental factors. 
In conclusion, medically assisted procreation has increased enormously in recent years [15]. Thus, in vitro fertilization or more recently ICSI (intracytoplasmic sperm injection) have allowed many couples unable to procreate naturally to have children. But these techniques, and especially their trivialization, pose certain problems. They allow overcoming the defect preventing a correct fertilization, but if this defect is of genetic nature, what is the risk of transmission to children born by medically assisted procreation? Although, in the majority of cases, the origin of the anomaly is not identified, many therapeutic means are available for infertile couples [15].

\section{References}

1. Roberts KP. Y-chromosome deletions and male infertility: state of the art and clinical implications. J Androl. 1998; 19:255-9.

2. Bhasin S, De Kretser DM, Baker HWG. Clinical review 64: pathophysiology and natural history of male infertility. J Clin Endocrinol Metab. 1994;79:1525-9.

3. Boué A, Novaes S. Rapport du groupe de travail sur l'assistance médicale à la procréation. Médecine/Sciences. 1994;10:929-35.

4. Haidl G. New aspects of the aetiology of male fertility disorders. Arch Gynecol Obset. 1994;255: S301-8.

5. Jégou B. Les hommes deviennent-ils moins fertiles? La Recherche. 1996;288:60-5.

6. Carlsen E, Giwercman A, Keiding N, et al. Evidence for decreasing quality of semen during past 50 years. Br Med J. 1992;305:609-13.
7. Auger J, Kunstmann JM, Czyglik F, et al. Decline in semen quality among fertile men in Paris during the past 20 years. N Engl J Med. 1995;332:281-5.

8. Rosenbusch BE. Cytogenetics of human spermatozoa: What about the reproductive relevance of structural chromosome aberrations? J Assist Reprod Genet. 1995;12:375-83.

9. Vogt PH. Genetic aspects of human infertility. Int J Androl. 1995; 18:3-6.

10. Dam AH, Koscinski I, Kremer JA, et al. Homozygous mutation in SPATA16 is associated with male infertility in human globozoospermia. Am J Hum Genet. 2007;81:81320.

11. Dieterich K, Soto Rifo R, Faure AK, et al. Homozygous mutation of AURKC yields large-headed polyploid spermatozoa and causes male infertility. Nat Genet. 2007;39:661-5.

12. Harbuz R, Zouari R, Pierre V, et al. A recurrent deletion of DPY19L2 causes infertility in man by blocking sperm head elongation and acrosome formation. Am J Hum Genet. 2011;88:351-61.

13. Koscinski I, Elinati E, Fossard C, et al. DPY19L2 deletion as a major cause of globozoospermia. Am J Hum Genet. 2011;88:344-50.

14. Ray PF. Deciphering the genetics of male infertility: Progress and challenges. J Urol. 2011;186:1183-4.

15. Morris RS, Gleicher N. Genetic abnormalities, male infertility, and ICSI. Lancet. 1996;347:1277.

\section{*Correspondence to:}

Ahmed Tabbabi

Department of Hygiene and Environmental Protection

Ministry of Public Health

Tunis

Tunisia

Tel: 0021697085424

E-mail: tabbabiahmed@gmail.com 